\title{
Doped Core Microstructured Fibers for Bragg Grating Sensor Applications
}

\author{
Tomasz Nasilowski ${ }^{*}$ \\ Department of Applied Physics and Photonics, Vrije Universiteit Brussel, Pleinlaan 2, B-1050 Brussels, Belgium
}

Received September 28, 2009; accepted September 30, 2009; published September 30, 2009

\begin{abstract}
The combination of a microstructured fiber (MSF) with fiber Bragg grating (FBG) is an architecture which joins two interesting ideas. On the one hand we have the Bragg grating, a device which has been thoroughly studied and successfully employed in numerous applications. On the other hand the photonic crystal fiber with its many, recently discovered possibilities. To a rather big extent we can consider such components as three dimensional photonic crystal structures. However, the exploitation of FBGs may require the use of MSFs with a doped core. An investigation of light propagation in a doped core MSF is presented. The conditions for the co-existence of two competing light guiding mechanisms (material and geometrical) and their consequences on the modes propagation are discussed.
\end{abstract}

One of very important and popular types of fiber sensors relies on fiber Bragg grating (FBG) [1][2]. FBG sensors are commonly used in civil engineering (bridges, dams, railways, mines etc). They consist (70\% to $80 \%$ ) of optical sensors in structural health monitoring.

Fiber Bragg gratings in conventional fibers have played a major role in many applications [3][4], and therefore in recent years there have been several attempts to incorporate a Bragg grating in an MSF through different techniques.

Despite their high maturity, classical fibers have limits in designing their properties. This might sometimes limit their applications or make them less competitive e.g. with electronic devices. This situation often took place in the case of sensing purposes and for that reason classical fiber sensors were mostly employed in niche applications.

Microstructured fibers consist of a periodic lattice of air holes that run along the length of the fiber and that allow confining and guiding light along the fiber. A defect in the centre of the lattice serves as a fiber core. Adapting particular features of the microstructure, such as the air filling fraction and the lattice period, allows to obtain optical fibers with very particular properties in terms of dispersion, mode-field confinement, singlemodedness, polarization etc. The optical and mechanical properties of MSFs can also be controlled by changing the size, shape and location of the air holes [5].

The unusual properties of an MSF deliver significantly

"E-mail: tnasilowski@tona.vub.ac.be improved performance in some respects to traditional fibers. They are also superior in one or few characteristics and eventually reveal the novel properties of fibers. All this leads to the superiority of implementations including sensing applications of MSFs [6][7].

Classical optical fibers are today finding wide use in areas covering telecommunications, sensor technology, spectroscopy, medicine etc [8]. Their operation usually relies on light being guided by the difference in material properties sometime called index guiding, and often mistaken with total internal reflection phenomena due to its intuitive properties taken from geometrical optics. Precisely, light in classical fibers is guided in the core region filled by the material with a higher refractive index and surrounded by the cladding region filled by the material with a lower refractive index. Some of the modes allowed by the Maxwell equations or wave equation [9] in the core are not allowed in the cladding region. In other words, some stationary solutions of the Maxwell equations in the higher refractive index material do not exist in the lower refractive index material. For this reason, if such modes are excited in the core region they have to propagate along the core down the length of the fiber since their existence is forbidden in the cladding. Because the propagation in classical fibers is steamed by the difference in the core and the cladding materials such a guiding mechanism can be called material guiding.

In the case of microstructured fibers the difference between the core and cladding is not in different material properties, but in different geometries of the same material. Due to this difference in geometry some of the modes are allowed in the core and are not allowed in the microstructured cladding. If these modes are excited in the core they are trapped over there and they can propagate only along the core. This is intuitively illustrated on the Fig.1A. Whenever the modes excited in the core are similar to the cladding modes they are not propagating because of the coupling to the cladding modes, as shown in Fig.1B. Since propagation is founded by the difference in geometries such a guiding mechanism can be called geometrical guiding. 
Geometrical guiding explains the propagation in both types of microstructured fibers, namely index guiding and photonic bandgap (PBG) PCFs.
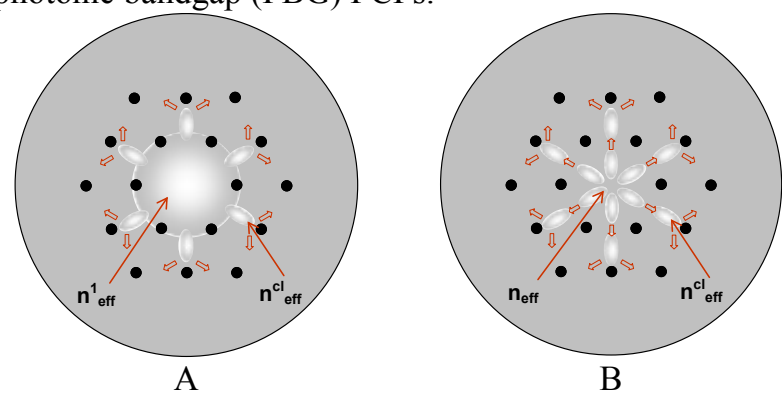

Fig. 1. Schematic drawing of a typical microstructured fiber presents guided (A) and not guided (B) modes. If the mode excited in the core has an effective refractive index $\left(\mathrm{n}^{1}\right.$ eff $)$ different from the effective index

of cladding modes $\left(\mathrm{n}^{\mathrm{cl}}\right.$ eff) it is trapped by the surrounding cladding structure and has to propagate along the core. In the case of the excited mode the core has a similar effective refractive index $\left(\mathrm{n}_{\text {eff }}\right)$ to the one of the cladding modes, it becomes the leaky mode and is coupled out from the core.

A typical index guiding PCF [10] consists of a solid glass core and a microstructured hollow cladding very often surrounded again by a larger solid glass region (Fig. 1). In this case the effective refractive index of the mode guided in the solid core is higher than the index of the microstructured cladding modes. For that reason this type of fiber is compared with classical, material guided fibers. Because of the great distinction of very high dispersion properties, due to the geometry scaled with wavelength, and selectivity of cladding modes in contrast with almost a continuum of modes in large and solid cladding of classical fiber there are remarkable differences which can not be neglected and are the basis for most of the extraordinary properties of PCFs.

Except for these more intuitive index guiding PCFs, within the past years the research in new microstructured materials has opened up the possibilities of localizing and controlling light in cavities and waveguides called the photonic bandgap (PBG) effect [11][12]. The propagation mechanism in the fiber with the PBG cladding is also based on geometrical guiding.

The large extent of freedom encoded in the complex geometry of an air-silica microstructure in the crossprofile of holey fibers can be used to enhance or optimize most of the important fiber properties. This includes the possibility of tailoring the modal structure, dispersion characteristics, birefringence or the mode area as well as following the strength of nonlinear effects.

MSFs, both index guiding and photonic bandgap guiding, constitute a new class of optical fibers which has revealed many interesting phenomena, paving the way for a large number of novel applications either in the telecom or in the sensing domain. However, some of the applications, especially including exploitation of FBGs, may require the use of specialty fibers with a doped core. An investigation of fundamental and higher order modes propagating in doped core MSF is presented in the next paragraphs focusing on the conditions for the coexistence of two competing light guiding mechanisms (material and geometrical) and their consequences on the modes propagation.

A typical MSF consists of pure silica with a periodic distribution of air holes in the cladding. However, some of the applications require the use of specialty fibres with a doped core. The latter plays either the role of active region in fibre lasers and amplifiers or that of an UVsensitive region for writing fibre Bragg gratings. Such a doped core MSF gives more opportunities for tailoring and designing the intensity distribution of the guided modes, which can be an asset of this type of fibre with respect to the classical doped core step index fibre (SIF).

Moreover, a doped core decreases the confinement and bending losses, and it allows using standard procedures and setups for FBG inscription. Generally speaking, one may influence the fibre properties by altering the holes diameter and distance, doping level and doped region diameter.

If we assume that the doped core MSF is a superposition of two different types of fibre, namely the MSF and the standard SIF (Fig. (2)), then we can combine two main guiding mechanisms, namely material and geometrical guiding into one fibre.

These two guiding mechanisms help us to explain the propagation regimes of the doped core MSF indicated in Fig. (2). The first regime (I) allows the propagation of only one mode through the geometrical guiding mechanism. Neither higher order MSF modes nor any SIF modes are allowed to propagate in that regime, though the propagated mode is slightly altered by the doped core. It is possible to modify the propagated mode distribution in order to improve the FBG coupling efficiency by carefully choosing the parameters of the doped core MSF within region (I). This regime is often preferred since it preserves all the extraordinary properties of geometrical guiding of an adequately designed MSF for a given application.

In the next regime (II), the material guiding mechanism is dominating and responsible for light propagation. The doped core MSF is still a single mode fibre, but most of the energy of the fundamental mode is contained within the doped core. In this case one may have the most efficient response of the Bragg grating confined in the photo-sensitive core and still be able to slightly modify the guiding conditions by adapting the MSF parameters. However, in this case the material guiding mechanism is dominating and most of the interesting properties of an MSF are vanished. The use of a microstructure in such a 
fibre should be reconsidered and double checked if it is really necessary.
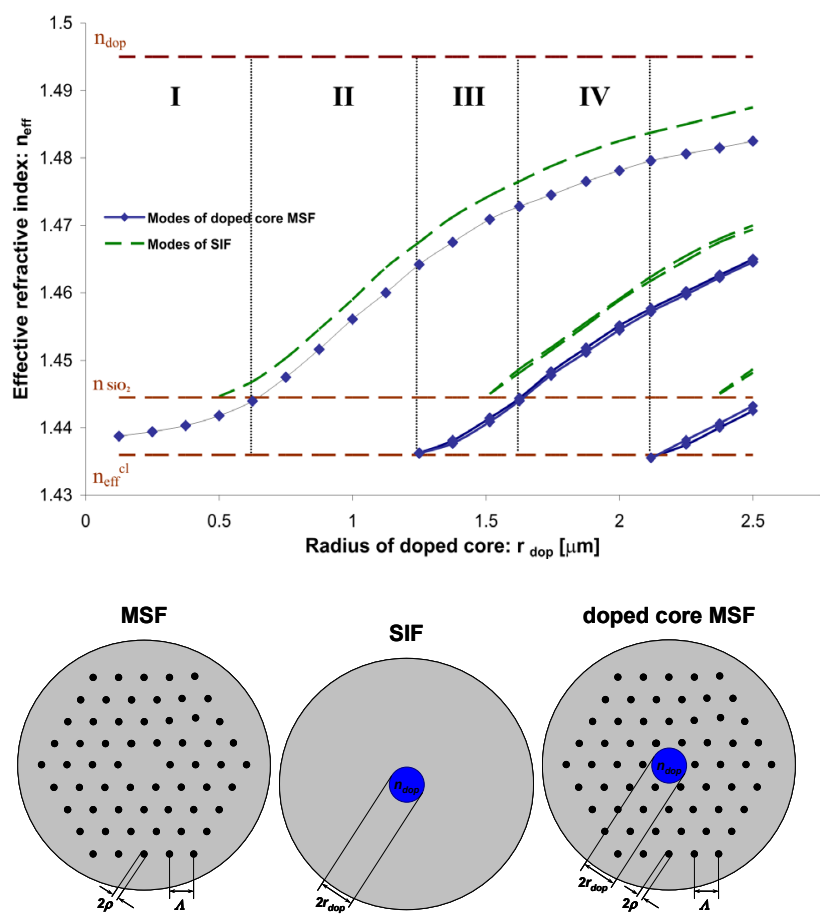

Fig. 2. Effective refractive indices of guided modes vs. radius of doped core for a given doping level (ndop) shown on the graph with a horizontal line. The solid curves represent modes guided in a doped core MSF and the dashed curves stand for modes propagated without a microstructure (modes of SIF). The two dashed horizontal lines represent respectively the refractive index of silica $(\mathrm{nSiO} 2)$ and the effective refractive index of the MSF cladding. The dotted vertical lines are the borders between four (I-IV) different propagation regimes of guided modes.

In regime (III) the two higher modes start to propagate. They propagate through the geometrical guiding mechanism, but at the same time the fundamental mode is propagated by the material guiding mechanism. This coexistence of both guiding methods brings forward very interesting differences in modes properties, especially those dispersion related. All fundamental mode properties are much less dependent on wavelength than the characteristics of higher order guided modes.

The fourth (IV) guiding regime consists of the fundamental and second order modes propagating within the doped core by the material mechanism, so most of the energy is concentrated in the doped core and the microstructure seems to be rather unnecessary.

Whenever a doped core MSF is considered, the strength of both guiding mechanisms (material or geometrical) must be verified. In case the material guiding becomes much stronger the geometrical guiding turns out to be negligible for light propagation and, practically, the microstructure does not influence any of the vital fibre properties. On the other hand, the presence of any doping region in the microstructured fibre does not exclude the crucial influence of the geometrical guiding mechanism on the parameters of such a fibre. It was demonstrated in [13] that a circular and highly Germanium doped MSF core (several mol\%) does not cancel very high birefringence caused by the pure geometrical effect of a microstructure.

The Bragg grating in a PCF may offer a viable alternative to traditional optical fibre sensors that require temperature compensation mechanisms and that are not intrinsically capable of distinguishing stress and temperature [14][15].

The unusual properties of MSFs deliver significantly improved performance in respect to traditional fibres. They are also superior in several characteristics and also unveil the novel properties of fibres. All this leads to the superiority of implementations and original applications of FBGs in MSFs.

The author would like to thank all partners contributing to the research on microstructured fibers, in particular all colleagues from Vrije Universiteit Brussel, Marie CurieSklodowska University, Wroclaw University of Technology and the Warsaw University of Technology. The work was partly supported by the EU FP7 project "PHOSFOS", the COST 299 action "FIDES" and the IWT SBO project "FAOS".

\section{References}

[1] K. O. Hill, Y. Fujii, D. C. Johnson, B. S. Kawasaki, Appl. Phys. Lett 1978; 32: 647-649.

[2] K. O. Hill, G. Meltz, J. Lightwave Technol. 1997; 15: 1263-1276.

[3] R. Kyashap Fiber Bragg Gratings. Academic Press 1999

[4] T. Erdogan, J. Lightwave Technol. 1997; 15: 1277-1294.

[5] P. St. J. Russell, J. Lightwave Technol. 2006; 24: 4729-4749.

[6] T. Nasilowski, F. Berghmans, T. Geernaert, et al. Proc. IEEE International Symposium on Intelligent Signal Processing 2007; WISP.2007.4447526: 1-6.

[7] T. Nasilowski, T. Martynkien, G. Statkiewicz, at al. J. Appl. Phys. B 81, pp. 325-331 (2005).

[8] A. Bjarklev Optical Fiber Amplifiers: Design and System Application. Artech House, Boston/London, 1993

[9] A. Snyder, J. Love Optical Waveguide Theory. Chapman \& Hall, London 1987.

[10] J. C. Knight, T. A. Birks, P. St. J. Russell, et al. Opt. Lett. 1996; 21: 1547.

[11] E. Yablonovitch, Phys. Rev. Lett. 1987; 58: 2059.

[12] A. Mekis, J. C. Chen, I. Kurland, et al. Phys. Rev. Lett. 1996; 77: 3787.

[13] T. Geernaert, G. Luyckx, E. Voet, et al. IEEE Photonics Technology Letters 2009; 21(1): 6-8.

[14] T. Geernaert, T. Nasilowski, K. Chah, et al. IEEE Photon. Technol. Lett. 2008; 20: 554-556.

[15] G. Luyckx, E. Voet, T. Geernaert, et al. IEEE Photonics Technology Letters 2009; 21(18): 1290-1292. 